\title{
On Sex-Related Differences in Auditory and Visual Sensory Functioning
}

\author{
Thomas H. Rammsayer · Stefan J. Troche
}

Received: 14 February 2011 / Revised: 27 October 2011 / Accepted: 19 November 2011 / Published online: 20 December 2011

(c) Springer Science+Business Media, LLC 2011

\begin{abstract}
The present study was designed to elucidate sexrelated differences in two basic auditory and one basic visual aspect of sensory functioning, namely sensory discrimination of pitch, loudness, and brightness. Although these three aspects of sensory functioning are of vital importance in everyday life, little is known about whether men and women differ from each other in these sensory functions. Participants were 100 male and 100 female volunteers ranging in age from 18 to 30 years. Since sensory sensitivity may be positively related to individual levels of intelligence and musical experience, measures of psychometric intelligence and musical background were also obtained. Reliably better performance for men compared to women was found for pitch and loudness, but not for brightness discrimination. Furthermore, performance on loudness discrimination was positively related to psychometric intelligence, while pitch discrimination was positively related to both psychometric intelligence and levels of musical training. Additional regression analyses revealed that each of three predictor variables (sex, psychometric intelligence, and musical training) accounted for a statistically significant portion of unique variance in pitch discrimination. With regard to loudness discrimination, regression analysis yielded a statistically significant portion of unique variance for sex as a predictor variable, whereas psychometric intelligence just failed to reach statistical significance. The potential influence of sex hormones on sex-related differences in sensory functions is discussed.
\end{abstract}

Keywords Sex differences - Pitch discrimination . Loudness discrimination $\cdot$ Brightness discrimination

T. H. Rammsayer $(\bowtie) \cdot$ S. J. Troche Department of Psychology, University of Bern, Muesmattstrasse 45, 3000 Bern 9, Switzerland e-mail: thomas.rammsayer@psy.unibe.ch

\section{Introduction}

Many researchers, including experimental psychologists and neurophysiologists, proceed from the assumption that most sensory functions are remarkably without sex-related differentiation (Baker, 1987). As a consequence, little attention has been paid to the consideration of sex differences in sensory functioning (cf. Ellis et al., 2008; Kimura, 1999). On the other hand, functional differences in sensory systems specialized in the reception and processing of environmental information may contribute to differences between men and women in their perception of the external world (cf. Mountcastle, 1975). If, in fact, men and women differ in their basic sensory experience, it is conceivable that such differences may be involved in the establishment of more complex sex-typed behaviors beyond sensory information processing (Baker, 1987; McGuinness, 1972; Velle, 1987). Given these considerations, investigating sex-related differences in sensory functions may represent a worthwhile endeavor. The present study, therefore, was designed to elucidate sex-related differences in two basic auditory and one basic visual aspect of sensory functioning, namely sensory discrimination of pitch, loudness, and brightness. Although these three aspects of sensory functioning are of vital importance in everyday life, to date, surprisingly little is known about whether men and women differ from each other in these sensory functions.

Early researchers in the late-nineteenth and early-twentieth centuries already investigated individual differences in the ability to make fine sensory discriminations. These early studies were inspired mainly by Galton's $(1869,1883)$ idea of a positive functional relationship between intelligence and performance on sensory discrimination. Moreover, Galton (1883) stated that "as a rule...men have more delicate powers of discrimination than women" (p. 20). This statement, however, was not based on experimental data and statistical analyses but on impressions and everyday-life anecdotes (cf. Deary, 1994). In his seminal paper 
on the measurement of general intelligence, Spearman (1904, Experiment 1) examined the relation between intelligence, as indicated by ranked school achievement of 13 girls and 11 boys, and performance on three sensory discrimination tasks: discrimination of pitch, brightness, and weight. His statistical analyses were based on a single measure derived from the general average correlation between the three forms of sensory discrimination performance, referred to as General Discrimination. Although General Discrimination and sex showed an initial correlation with one another to the extent of $r_{x y}=.26$, after controlling for intelligence, this correlation decreased to $r_{x y}=-.07$. This led Spearman to conclude that sex-related differences in general discrimination can be considered a mere consequence of individual differences in intelligence.

Other studies on sex-related differences in sensory discrimination focused on more specific functions rather than on General Discrimination. In the following, we will provide a brief account of some major studies on sex-related differences in pitch, loudness, and brightness discrimination.

\section{Pitch Discrimination}

The few existing studies on sex-related differences in pitch discrimination in adults present a rather puzzling picture of inconsistent results. With 29 male and 19 female young adult participants, Seashore (1899) found better performance in women than in men. For Seashore, the ability to detect differences in pitch was a fundamental factor in the appreciation or execution of music. Therefore, he assumed that higher levels of musical education in women compared to men accounted for this female superiority. On the other hand, Shuter (1968) reported slightly superior performance on pitch discrimination for men compared to women, while men and women did not appear to be very different in the frequently cited study by McGuinness (1972). In this latter study, however, musical training in terms of years spent on any instrument was reliably related to better pitch discrimination. In a subsequent study by Raz, Willerman, and Yama (1987), there was no effect of sex on pitch discrimination, and musical training failed to show a significant association with pitch discrimination. It should be noted, however, that, in this study, extremely brief tones were applied lasting $20 \mathrm{~ms}$ only. In addition, there was a constant background noise of at least $35 \mathrm{~dB}$ throughout the experiment. These peculiarities cast some doubt on whether the measures obtained by Raz et al. represent an indicator of performance on genuine pitch discrimination.

\section{Loudness Discrimination}

Several studies confirmed superior auditory sensitivity, as reflected by pure tone thresholds, in women compared to men (for a concise review, see Baker, 1987). In these studies, auditory sensitivity was typically measured by determining the absolute threshold for detection of a sound which is the lowest amplitude (i.e., volume) an individual was able to perceive. In the light of these highly consistent findings with regard to pure tone thresholds, it is most surprising that virtually no studies appear to exist on sex-related differences in the ability to perceive differences in the loudness of different tones referred to as loudness discrimination (cf. Baker, 1987; Ellis et al., 2008; Kimura, 1999; Maccoby \& Jacklin, 1974; Velle, 1987).

To the best of our knowledge, the first and only psychophysical investigation that directly addressed the issue of sex-related individual differences in loudness discrimination was conducted by Seashore (1919). He obtained least perceptible differences in the intensity, or loudness, of a standard tone from 522 seventh and eighth graders, but did not report any sex-related differences in his studies on "The Psychology of Musical Talent”. He also addressed the possible relationship between loudness discrimination and intelligence: "This test [loudness discrimination] has often been used as an intelligence test; but that cannot be justified because we find very little agreement between excellence in this test and brightness of children as ordinarily estimated" (Seashore, 1919, p. 98). To date, data on sex-related differences in loudness discrimination still seem to be missing for adults.

\section{Brightness Discrimination}

In his pioneering studies on the mental and physical development of schoolchildren from 6 to 17 years of age, Gilbert (1894) measured least perceptible differences in shade of the color red. Although the color gray would have been preferable to red for the assessment of brightness discrimination in order to avoid a confound of general brightness discrimination with color sensitivity, Gilbert felt compelled to use red due "to the fact that all goods are bleached with sulphur (and), no matter how well scoured before dyeing, traces of red could be found running through the gray" (Gilbert, 1894, p. 42). With this procedure, girls tended to outperform boys in brightness discrimination. Gilbert (1894), however, refrained from making a final statement as to comparison of sexes since performance curves of boys and girls crossed and re-crossed frequently as a function of age. Also, our reanalysis of Spearman's (1904) data based on 24 schoolchildren gave no indication of sex-related differences in brightness discrimination after controlling for individual differences in intelligence. Strangely enough, however, there seems to be no information on performance differences between male and female adults (cf. Baker, 1987; Ellis et al., 2008; Kimura, 1999; Maccoby \& Jacklin, 1974; Velle, 1987).

Proceeding from these considerations, the major focus of the present study was on elucidating sex-related differences in performance on pitch, loudness, and brightness discrimination. For this purpose, 100 male and 100 female adults were tested under controlled conditions. Since sensory sensitivity may be positively related to individual levels of intelligence (e.g., Acton \& Schroeder, 2001; Burt, 1909-1910; Deary, Bell, Bell, Campbell, \& Fazal, 2004; Raz, Moberg, \& Millman, 1990; Raz et al., 
1987; Schweizer \& Koch, 2003; Troche \& Rammsayer, 2009) and musical experience (Seashore, 1919), measures of psychometric intelligence and musical background were also obtained.

\section{Method}

Participants

Participants were 100 male and 100 female volunteers ranging in age from 18 to 30 years (mean and $S D$ of age: $22.2 \pm 3.3$ years). Each group consisted of 50 student and 50 non-student participants. Participants were recruited by announcements posted at notice boards on campus as well as in adult education and community centers. All participants reported normal hearing and normal or corrected-to-normal sight. They were informed about the study protocol and gave their written informed consent. For taking part in this study, participants were paid EUR 30.00 and feedback was offered about their performance on intelligence testing.

\section{Measures and Procedure}

\section{Sensory Discrimination Tasks}

For quantification of individual discrimination performance, an adaptive psychophysical procedure, the weighted up-down method (Kaernbach, 1991), was applied. "Adaptive" means that the difference in stimulus magnitude between the constant standard stimulus and the variable comparison stimulus, presented within a trial, is varied from trial to trial depending on the participant's previous response. Correct responding results in a decrease of the difference in stimulus magnitude and incorrect responding makes the task easier by increasing this difference. With this psychophysical procedure, $x .25$ and $x .75$ of the individual psychometric function were estimated. $x .25$ and $x .75$ refer to the two levels of stimulus magnitude of the comparison stimulus at which the response "higher" (pitch discrimination), "louder" (loudness discrimination) or "brighter" (brightness discrimination) was given with a probability of .25 and .75 , respectively.

On each sensory task, one series of 32 trials converging to $x .25$ and one series of 32 trials converging to $x .75$ were presented. Within each 32-trial series, the order of presentation for the standard and the comparison stimulus was randomized and balanced, with each stimulus being presented first in $50 \%$ of the trials. Trials from both series were randomly interleaved.

To initiate a trial, the participant pressed the space bar; stimulus presentation began $900 \mathrm{~ms}$ later. The two stimuli were presented successively with an interstimulus interval of $300 \mathrm{~ms}$. The participant's task was to decide which of the two stimuli was greater in magnitude and to indicate his or her decision by pressing one of two designated keys on the keyboard. Instruction emphasized accuracy; there was no requirement to respond quickly. After each response, visual feedback ("+"= correct or "-" = false) was displayed for $1,000 \mathrm{~ms}$ on the monitor screen. The next trial started $900 \mathrm{~ms}$ after the offset of the visual feedback.

Mean differences between levels of magnitude of the standard and comparison stimulus were computed for the last 20 trials of each series. Thus, estimates of $x .25$ and $x .75$ were obtained. As an indicator of discrimination performance, half the interquartile ranges $[(x .75-x .25) / 2]$, representing the just noticeable difference or difference limen, DL (Luce \& Galanter, 1963), were determined for all three sensory discrimination tasks. With this psychophysical measure, better sensory discrimination was indicated by smaller DL values.

\section{Pitch Discrimination (PD)}

Stimuli were 500-ms sine-wave tones presented through headphones (SONY MDR-CD570) at an intensity of $67 \mathrm{~dB}$. On each trial, participants had to decide which of two tones was of higher pitch. The pitch of the constant standard tone was $440 \mathrm{~Hz}$. The initial comparison stimulus was $438 \mathrm{~Hz}$ in the $x .25$ series and $442 \mathrm{~Hz}$ in the $x .75$ series. In the first six trials of the $x .25$ series, pitch of the comparison stimulus was increased by $0.5 \mathrm{~Hz}$ and decreased by $1.5 \mathrm{~Hz}$ after a correct or incorrect response, respectively. For Trials 7-32, step sizes were 0.3 and $0.9 \mathrm{~Hz}$, respectively. Opposite step sizes were used for the $x .75$ series.

\section{Loudness Discrimination (LD)}

Stimuli were white-noise bursts presented through headphones for $500 \mathrm{~ms}$. Participants had to decide which of two noise bursts was louder. The intensity of the standard tone was $70 \mathrm{~dB}$, the intensity of the initial comparison stimulus was $69.70 \mathrm{~dB}$ in the $x .25$ series and $70.30 \mathrm{~dB}$ in the $x .75$ series. In the first six trials of the $x .25$ series, intensity of the comparison stimulus was increased by $.07 \mathrm{~dB}$ and decreased by $.21 \mathrm{~dB}$ after a correct or incorrect response, respectively. For Trials 7-32, step sizes were .02 and $.06 \mathrm{~dB}$, respectively. Opposite step sizes were used for the $x .75$ series.

\section{Brightness Discrimination (BD)}

Visual stimuli were grey rectangles (all three RGB settings were equal) on a white background subtending $16.5^{\circ}$ of visual angle vertically and horizontally, respectively. On each trial, two stimuli were presented successively for $800 \mathrm{~ms}$ in the center of a 19" TFT-monitor screen (ViewSonic VS10162). The participant's task was to decide whether the first or the second rectangle was brighter. Brightness of the standard stimulus was $32.7 \mathrm{~cd} / \mathrm{m}^{2}$. Brightness of the initial comparison stimulus was $35.7 \mathrm{~cd} / \mathrm{m}^{2}$ in the $x .25$ series and $29.7 \mathrm{~cd} / \mathrm{m}^{2}$ in the $x .75$ series. In 
the first six trials of the $x .25$ series, brightness of the comparison stimulus was increased by $0.4 \mathrm{~cd} / \mathrm{m}^{2}$ and decreased by $1.2 \mathrm{~cd} / \mathrm{m}^{2}$ after a correct and incorrect response, respectively. For Trials 732 , step sizes were 0.3 and $0.9 \mathrm{~dB}$, respectively. Opposite step sizes were employed for the $x .75$ series.

\section{Psychometric Intelligence}

For the measurement of psychometric intelligence, subtests of the Berlin Model of Intelligence Structure (BIS) test (Jäger, Süß, \& Beauducel, 1997) were administered. This test is a paperpencil test based on Jäger's (1984) BIS model of intelligence. The BIS model classifies cognitive abilities with respect to the required mental operations (reasoning, speed of processing, memory, and creativity) and, concurrently, with respect to the contents of the processed tasks (verbal, numerical, and figural contents). Thus, the BIS subtests are combinations of operations and contents (e.g., reasoning for processing verbal, numerical, and figural contents). A more detailed description of the BIS model and an evaluation of the BIS test is provided by Bucik and Neubauer(1996) as well as Süß, Oberauer, Wittmann, Wilhelm, and Schulze (2002).

For the present study, six reasoning and six speed tests with two subtests for each content (verbal, numerical, figural) were used. The BIS model does not consider contents and operations as independent dimensions so that a general factor of intelligence has been assumed (Jäger et al., 1997). General intelligence, referred to as psychometric $g$, was quantified as the first unrotated component of a principal component analysis across all $z$-standardized subtest measures (cf. Jensen, 1998; Jensen \& Reynolds, 1982). With this procedure, higher factor scores indicate higher levels of psychometric $g$.

\section{Musical Background}

Years of musical training were recorded on a 3-point rating scale: (1) have never played a musical instrument, (2) have less than five years of musical training, and (3) have five or more years of musical training.

\section{Time Course of the Study}

The intelligence tests and the experimental tasks were applied in two different sessions with a 1-week interval between the sessions. For half of the participants, the sensory tasks were preceded by psychometric assessment of intelligence, while for the other half, intelligence tests were administered in the first session. Psychophysical assessment of sensory discrimination performance took place in a sound-attenuated room with constant ambient light. Order of experimental tasks was counterbalanced across participants.

\section{Results}

Table 1 reports mean extent of musical training, mean factor scores on psychometric $g$, and mean performance on the three sensory discrimination tasks for male and female participants. As can also be seen in Table $1, t$-tests revealed that psychometric intelligence and performance on pitch and loudness discrimination was significantly better for the men than for the women. Significant differences between both groups were shown neither for the extent of musical training nor for brightness discrimination.

To illustrate the significance of the observed effect size indices of $d=.62$ and $d=.40$ for pitch and loudness discrimination, respectively, $d$ can be converted into percentage of nonoverlap (Cohen, 1988). When $d=0$, the distribution of performance scores (expressed as the DL) for the male and the female group would be perfectly superimposed on each other, i.e., there is $100 \%$ overlap. Based on this consideration, an effect size $d=$ .62 for pitch discrimination means that $39.2 \%$ of the area covered by the male and female group combined is not overlapped (cf. Cohen, 1988). Similarly, the effect size $d=.40$ for loudness discrimination indicates a non-overlap of $27.4 \%$, i.e., $27.4 \%$ of the combined area was not shared by the sexes.

Additional correlational analyses revealed that performance on all three sensory discrimination tasks was positively related to psychometric intelligence. There also was a reliable positive correlation between extent of musical training and performance on pitch discrimination, while no such association could be observed for loudness and brightness discrimination (see Table 2).

In a next step, the fact that performance on all three sensory discrimination tasks was positively related to individual levels of psychometric intelligence was accounted for by performing additional one-way analyses of covariance with psychometric $g$ as a covariate. Because performance on pitch discrimination

Table 1 Means and SEM for musical background, psychometric intelligence, and performance on the three sensory discrimination tasks for male $(N=100)$ and female $(N=100)$ participants

\begin{tabular}{|c|c|c|c|c|c|c|}
\hline & \multicolumn{2}{|c|}{ Males } & \multicolumn{2}{|c|}{ Females } & \multirow[t]{2}{*}{$t$} & \multirow[t]{2}{*}{$d$} \\
\hline & $M$ & SEM & $M$ & SEM & & \\
\hline Musical background & 1.78 & .08 & 1.99 & .08 & 1.94 & .27 \\
\hline Psychometric intelligence & .23 & .10 & -.23 & .09 & $-3.33 * * *$ & .47 \\
\hline Pitch (DL in $\mathrm{Hz})$ & 3.69 & .20 & 4.99 & .22 & $4.38 * * *$ & .62 \\
\hline Loudness (DL in $\mathrm{dB}$ ) & .57 & .02 & .64 & .02 & $2.86^{* *}$ & .40 \\
\hline Brightness (DL in $\mathrm{cd} / \mathrm{m}^{2}$ ) & 3.94 & .12 & 4.01 & .14 & .37 & .0 \\
\hline
\end{tabular}

Significantly higher factor scores indicate a higher level of psychometric intelligence for the male compared to the female group. Significantly smaller DLs indicate better pitch and loudness discrimination for men compared to women

$* * p<.01 ; * * * p<.001$ (two-tailed) 
was also associated with individual musical background, the extent of musical training was introduced as a second covariate for the statistical analysis of pitch discrimination.

In this latter case, a statistically significant effect of sex, $F(1$, 196) $=17.77, p<.001$, partial $\eta^{2}=.08$, indicated better performance on pitch discrimination for males compared to females; adjusted mean DLs $( \pm$ SEM) were $3.73 \pm 0.078 \mathrm{~Hz}$ and $4.95 \pm$ $0.080 \mathrm{~Hz}$ for the male and female group, respectively.

Analysis of covariance controlling for individual levels of psychometric intelligence also yielded reliably better male performance on loudness discrimination, $F(1,197)=5.64, p<.05$, partial $\eta^{2}=.028$; adjusted mean DLs were $0.58 \pm 0.003$ and $0.64 \pm 0.002 \mathrm{~dB}$ for men and women, respectively. There was, however, no evidence for a sex-related difference in brightness discrimination, $F(1,197)<1$, partial $\eta^{2}=.003$. Thus, statistical removal of the effects of psychometric intelligence, in the case of loudness and brightness discrimination, or of the effects of both psychometric intelligence and musical training, in the case of pitch discrimination, did not alter the initial results obtained by $t$-tests.

To further elucidate the contributions of sex, psychometric intelligence, and musical background to individual differences in pitch discrimination performance, a regression analysis was calculated. The standardized beta weights were $\beta=-.28$ ( $p<$ $.001)$ for sex, $\beta=-.23(p<.001)$ for psychometric intelligence, and $\beta=-.25(p<.001)$ for musical background. All three predictors together explained a portion of $21.6 \%$ of overall variability in pitch discrimination. As indicated by semi-partial correlations, unique portions of variance of 7.1, 5.2, and 4.8\% (all $p$ values $<.001$ ) were accounted for by sex, psychometric intelligence, and musical background, respectively.

Loudness discrimination performance showed a significant sex difference as well as a significant positive association to psychometric intelligence. When considering both sex and psychometric intelligence in a regression analysis to predict performance on loudness discrimination, the standardized beta weights were $\beta=-.17(p<.05)$ for sex and $\beta=-.13(p=.07)$ for psychometric intelligence. Both predictors together explained 5.6\% of variance of loudness discrimination. Portions of variance uniquely explained by sex and psychometric intelligence were $2.6 \%(p<.05)$ and $1.7 \%(p=.07)$, respectively.

Table 2 Intercorrelations among psychometric intelligence, musical background, and performance on the three sensory discrimination tasks

\begin{tabular}{lllll}
\hline & $\begin{array}{l}\text { Musical } \\
\text { background }\end{array}$ & $\begin{array}{l}\text { Psychometric } \\
\text { intelligence }\end{array}$ & Pitch & Loudness \\
\hline $\begin{array}{l}\text { Psychometric } \\
\text { intelligence }\end{array}$ & .13 & & & \\
Pitch & $.24^{* *}$ & $.33^{* * *}$ & & \\
Loudness & .01 & $.17^{*}$ & $.22^{* *}$ & \\
Brightness & .06 & $.33^{* * *}$ & $.38^{* * *}$ & $.28 * * *$ \\
\hline
\end{tabular}

$* p<.05 ; * * p<.01 ; * * * p<.001$ (two-tailed)

\section{Discussion}

The present study investigated sex-related differences in the discrimination of pitch, loudness, and brightness as three basic aspects of sensory functioning. Reliably better performance for men compared to women was revealed for pitch and loudness discrimination. In a meta-analysis of research on psychological gender differences, Hyde (2005) arrived at the conclusion that, in terms of effect sizes, most sex-related differences are in the close-to-zero or small range (i.e., $0<d<.35$ ), while only few are in the moderate or large range $(d>.35)$. Thus, the effect sizes of $d=.62$ and $d=.40$ obtained in the present study for pitch and loudness discrimination, respectively, can be considered substantial. In Hyde's review, only very few cognitive variables, such as spatial perception $(d=.44)$ and mental rotation $(d$ ranging from .56 to .73 ), showed effect size estimates of a similar magnitude while, for the majority of all cognitive variables reviewed by Hyde (2005), effect size estimates were markedly smaller. Unlike discrimination of pitch and loudness, no significant difference between the sexes was found for brightness discrimination. Furthermore, performance on loudness discrimination was positively related to psychometric intelligence, while pitch discrimination was positively related to both psychometric intelligence and individual levels of musical training.

In his classic paper, Spearman (1904) argued that sex-related differences in sensory discrimination were mediated by different levels of intelligence of the male and female groups to be compared. Thus, when differences in mental ability were statistically removed before examining the relationship between sex and sensory discrimination, no sex-related performance differences should be found. In the present study, sex-related differences in pitch and loudness discrimination could be established. With both discrimination tasks, men performed reliably better than women. Contrary to Spearman's (1904) assumption, this finding held even after controlling for individual differences in general intelligence, in the case of loudness discrimination, and after controlling for individual differences in both general intelligence and musical training, in the case of pitch discrimination.

It is worth mentioning that the pattern of sex-related differences observed in the present study also suggests that Spearman's (1904) conception of a latent variable referred to as General Discrimination was not an appropriate approach for investigating sex-related differences in sensory discrimination. Rather, our finding of sex-related differences for pitch and loudness discrimination and the concurrent absence of such a difference for brightness discrimination indicate that sex-related differences in sensory discrimination depend upon the specific sensory function under investigation. Thus, sex-related differences in sensory discrimination cannot be considered a characteristic trait common to all aspects of sensory functioning.

In the present study, performance on pitch and loudness discrimination was positively associated with psychometric intelligence (as well as with musical training in the case of pitch 
discrimination). Therefore, additional regression analyses were performed to elucidate the unique contributions of sex, psychometric intelligence, and, in the case of pitch discrimination, musical background to the explanation of the observed overall variability in discrimination performance of both these sensory tasks. All three predictor variables had statistically significant unique contributions to account for overall variability in pitch discrimination. As the most powerful predictor variable, sex accounted for $7.1 \%$ of the variance followed by psychometric intelligence and musical training that accounted for portions of 5.2 and $4.8 \%$, respectively. This pattern of results suggests that individual differences in mental ability and musical background, if not controlled for, may produce a confounding effect that could mask a potential effect of sex on pitch discrimination. This may be a possible reason why McGuinness (1972) failed to observe sex-related individual difference in pitch discrimination. Although McGuinness (1972) found that years of musical training was reliably related to better pitch discrimination, she did not statistically control for the influence of musical background on pitch discrimination. Moreover, she did not measure psychometric intelligence to control for its influence on sexrelated differences in pitch discrimination. Therefore, it is conceivable that, in her study, potential influences of sex, musical background, and mental ability on pitch discrimination interfered with each other.

With regard to loudness discrimination, regression analysis yielded a statistically significant portion of unique variance of $2.6 \%$ for sex as predictor variable, whereas the portion of $1.7 \%$ of unique variance for psychometric intelligence just failed to reach the 5\%-level of statistical significance. Thus, our findings suggest much more potent, specific (unique) influences of sex, mental ability, and musical background on pitch than on loudness discrimination. Unlike pitch and loudness discrimination, there was no indication for a sex-related difference in performance on brightness discrimination.

Numerous studies on sex-related differences in auditory sensitivity as assessed by pure tone thresholds suggest lower absolute thresholds for the detection of a sound for women compared to men, particularly at higher frequencies (cf. Baker, 1987; Corso, 1959; Dreisbach, Kramer, Cobos, \& Cowart, 2007; Sax, 2010). The present study expands this finding by demonstrating a male advantage in the ability to perceive differences in the loudness of two tones. At first sight, the former finding of better performance on pure tone detection for women and the present finding of better loudness discrimination for men may appear contradictory. There are, however, at least two reasonable explanations that can account for these apparently inconsistent results.

First, from a psychophysical perspective, absolute judgments for estimating detection thresholds and comparison judgments for estimating difference thresholds may involve qualitatively different modes of cognitive processing (Nahum, Daikhin, Lubin, Cohen, \& Ahissar, 2010). Second, and even more important, there are distinct aspects of auditory sensitivity that are functionally independent of each other. Therefore, the direction of sex-related differences in auditory sensitivity is not consistent but varies as a function of the specific sensory task applied. As already mentioned, women outperform men in pure tone detection tasks (cf., Baker, 1987). On the other hand, superior male auditory sensitivity has been demonstrated, for example, with auditory masking tasks (Neff, Kessler, \& Dethlefs, 1996) and for the discrimination of small differences in interaural arrival times, i.e., differences in the time of arrival of a tone at the two ears (Langford, 1994). Most interestingly, in this latter study, Langford (1994) also showed that men were better than women at detecting interaural differences in sound intensity (see also McFadden, 1998). This male advantage in interaural sensitivity for sound intensity corresponds nicely to the present finding of better performance on binaural loudness discrimination for men compared to women. Given these considerations, future studies should be designed to further investigate both these tentative explanations.

In a review on sex differences in sensory functions, Velle (1987) concluded that differential sensitivity of the male and female auditory system may be influenced by sex hormones. Converging evidence for this hypothesis comes from studies on organizational and activational effects of sex hormones on measures of auditory sensitivity, such as auditory brain-stem responses, otoacoustic emissions or pure tone thresholds (e.g., McFadden, 2002, 2009). While organizational effects refer to permanent structural changes initiated primarily by prenatal hormone exposure, activational effects relate to the response elicited by the presence of a hormone after differentiation and organization (cf. Arnold \& Breedlove, 1985). Thus, activational effects occur at a later stage than organizational effects, usually during adulthood, and include normal cyclical physiological functions, such as the menstrual cycle. Furthermore, Velle (1987) pointed out that there seems to be no evidence for an influence of sex hormones on sensitivity of the visual system.

Such a conclusion appears to be consistent with the outcome of the present study. Sex-related differences could be established for pitch discrimination and loudness discrimination, two sensory functions of the auditory system, but not for brightness discrimination representing an aspect of visual acuity. Within Velle's (1987) biological framework, it is likely that sex-related individual differences in aspects of auditory sensitivity are the result of differences in the genetic program of men and women. Sex-related genetic influences are well-known to control the secretion of sex hormones, such as estrogens and androgens, during fetal life as well as after reaching puberty (e.g., Hines, 2009; Schulz, Molenda-Figueira, \& Sisk, 2009). Organizational effects of sex hormones during prenatal development or during puberty and activational effects after maturation might have contributed to the observed sex-related individual differences in pitch and loudness discrimination. Given Velle's (1987) biological line of reasoning, no such hormonal influence appears to become effective for visual sensory sensitivity and, thus, no sex- 
related differences are to be expected. This, however, does not necessarily mean that there are no sex-related differences with regard to other aspects of visual perception such as visual acuity, visual persistence or color blindness (cf. Ellis et al., 2008).

To sum up, the present study showed sex-related differences in pitch and loudness discrimination with men performing better than women. These differences remained stable even when controlling for individual levels of mental ability. Additional regression analyses revealed that each of the three predictor variables of sex, psychometric intelligence, and musical training accounted for a statistically significant portion of unique variance in pitch discrimination. Unlike pitch and loudness discrimination, as two aspects of auditory sensory functions, no sex-related differences could be observed for brightness discrimination. Although the ultimate cause for differences between men and women in the sensory functions investigated in the present study is still unknown, action of steroidal hormones may play a crucial role in the formation of these sex-related differences.

\section{References}

Acton, G. S., \& Schroeder, D. H. (2001). Sensory discrimination as related to general intelligence. Intelligence, 29, 263-271.

Arnold, A. P., \& Breedlove, S. M. (1985). Organizational and activational effects of sex steroids on brain and behavior: A reanalysis. Hormones and Behavior, 19, 469-498.

Baker, M. A. (1987). Sensory functioning. In M. A. Baker (Ed.), Sex differences in human performance (pp. 5-36). Chichester, England: Wiley.

Bucik, V., \& Neubauer, A. C. (1996). Bimodality in the Berlin model of intelligence structure (BIS): A replication study. Personality and Individual Differences, 21, 987-1005.

Burt, C. (1909-1910). Experimental tests of general intelligence. British Journal of Psychology, 3, 94-177.

Cohen, J. (1988). Statistical power analysis for the behavioral sciences. Hillsdale, NJ: Lawrence Erlbaum Associates.

Corso, J. F. (1959). Age and sex differences in thresholds. Journal of the Acoustical Society of America, 31, 489-507.

Deary, I. J. (1994). Sensory discrimination and intelligence: Postmortem or resurrection? American Journal of Psychology, 107, 95-115.

Deary, I. J., Bell, P. J., Bell, A. J., Campbell, M. L., \& Fazal, N. D. (2004). Sensory discrimination and intelligence: Testing Spearman's other hypothesis. American Journal of Psychology, 117, 1-18.

Dreisbach, L. E., Kramer, S. J., Cobos, S., \& Cowart, K. (2007). Racial and gender effects on pure-tone thresholds and distortion-product otoacoustic emissions (DPOAEs) in normal-hearing young adults. International Journal of Audiology, 46, 419-426.

Ellis, L., Hershberger, S., Field, E., Wersinger, S., Pellis, S., Geary, D., ... Karadi, K. (2008). Sex differences: Summarizing more than a century of scientific research. New York: Psychology Press.

Galton, F. (1869). Hereditary genius: An inquiry into its laws and consequences. London: Macmillan.

Galton, F. (1883). Inquiries into human faculty and its development. London: Macmillan.

Gilbert, J. A. (1894). Researches on the mental and physical development of school-children. Studies from the Yale Psychological Laboratory, 2, 40-100.

Hines, M. (2009). Gonadal hormones and sexual differentiation of human brain and behavior. In D. Pfaff, A. P. Arnold, A. M. Etgen, S.
E. Fahrbach, \& R. T. Rubin (Eds.), Hormones, brain and behavior (Vol. 4, pp. 1869-1909). New York: Academic Press.

Hyde, J. S. (2005). The gender similarities hypothesis. American Psychologist, 60, 581-592.

Jäger, A. O. (1984). Intelligenzstrukturforschung: Konkurrierende Modelle, neue Entwicklungen, Perspektiven [Research in the structure of intelligence: Competing models, new developments, perspectives]. Psychologische Rundschau, 35, 21-35.

Jäger, A. O., Süß, H.-M., \& Beauducel, A. (1997). Berliner Intelligenzstruktur test form 4. Göttingen: Hogrefe.

Jensen, A. R. (1998). The g factor. Westport, CT: Praeger Publishers.

Jensen, A. R., \& Reynolds, C. R. (1982). Race, social class and ability patterns on the WISC-R. Personality and Individual Differences, 3 , 423-438.

Kaernbach, C. (1991). Simple adaptive testing with the weighted up-down method. Perception \& Psychophysics, 49, 227-229.

Kimura, D. (1999). Sex and cognition. Cambridge, MA: MIT Press.

Langford, T. L. (1994). Individual differences in sensitivity to interaural disparities of time and level. Journal of the Acoustical Society of America, 96(Suppl. 1), 3256.

Luce, R. D., \& Galanter, E. (1963). Discrimination. In R. D. Luce, R. R. Bush, \& E. Galanter (Eds.), Handbook of mathematical psychology (Vol. 1, pp. 191-243). New York: Wiley.

Maccoby, E. E., \& Jacklin, C. N. (1974). The psychology of sex differences. Stanford, CA: Stanford University Press.

McFadden, D. (1998). Sex differences in the auditory system. Developmental Neuropsychology, 14, 261-298.

McFadden, D. (2002). Masculinization effects in the auditory system. Hearing Research, 252, 37-48.

McFadden, D. (2009). Masculinization of the mammalian cochlea. Archives of Sexual Behavior, 31, 99-111.

McGuinness, D. (1972). Hearing: Individual differences in perceiving. Perception, 1, 465-473.

Mountcastle, V. B. (1975). The view from within: Pathways to the study of perception. Johns Hopkins Medical Journal, 136, 109-131.

Nahum, M., Daikhin, L., Lubin, Y., Cohen, Y., \& Ahissar, M. (2010). From comparison to classification: A cortical tool for boosting perception. Journal of Neuroscience, 30, 1128-1136.

Neff, D. L., Kessler, C. J., \& Dethlefs, T. M. (1996). Sex differences in simultaneous masking with random-frequency maskers. Journal of the Acoustical Society of America, 100, 2547-2550.

Raz, N., Moberg, P. J., \& Millman, D. (1990). Effects of age and agerelated differences in auditory information processing on fluid and crystallized intelligence. Personality and Individual Differences, $11,1147-1152$.

Raz, N., Willerman, L., \& Yama, M. (1987). On sense and senses: Intelligence and auditory information processing. Personality and Individual Differences, 8, 201-210.

Sax, L. (2010). Sex differences in hearing. Implications for best practice in the classroom. Advances in Gender and Education, 2, 13-21.

Schulz, K. M., Molenda-Figueira, H. A., \& Sisk, C. L. (2009). Back to the future: The organizational-activational hypothesis adapted to puberty and adolescence. Hormones and Behavior, 55, 597-604.

Schweizer, K., \& Koch, W. (2003). Perceptual processes and cognitive ability. Intelligence, 31, 211-235.

Seashore, C. E. (1899). Some psychological statistics. In G. T. Patrick (Ed.), The University of Iowa studies in psychology (pp. 1-84). Iowa City, IA: The University of Iowa.

Seashore, C. E. (1919). The psychology of musical talent. Boston, MA: Silver, Burdett and Company.

Shuter, R. (1968). Psychology of music. London: Methuen.

Spearman, C. (1904). "General intelligence", objectively determined and measured. American Journal of Psychology, 15, 201-293.

Süß, H.-M., Oberauer, K., Wittmann, W. W., Wilhelm, O., \& Schulze, R. (2002). Working- memory capacity explains reasoning ability-and a little bit more. Intelligence, 30, 261-288. 
Troche, S. J., \& Rammsayer, T. H. (2009). Temporal and non-temporal sensory discrimination and their predictions of capacity- and speed-related aspects of psychometric intelligence. Personality and Individual Differences, 47, 52-57.
Velle, W. (1987). Sex differences in sensory functions. Perspectives in Biology and Medicine, 30, 490-522. 